\title{
NOTE ON PSEUDARTHROSIS OF THE TIBIA IN CHILDHOOD
}

\author{
A Discussion Arising from the Preceding Paper, with some \\ General Observations on the Healing of Fractures
}

\author{
A. N. Birkett, Nottingham, England
}

It is with some diffidence that I add observations to the subject that McFarland has made his own, but my genuine excuse is related to the hours of thought and work that have been provoked by the case he has kindly included in his series (Case 4, page 40). After the case had been discussed with him, a further event took place. Although firm union had been secured, the girl refractured her leg at the junction of the graft and lower third of the tibia, and despite immobilisation it proceeded towards non-union (Fig. 1). It seems possible that persistence of the pseudarthrosis might have arisen from factors that have not previously been discussed fully. Why, in this case, did the double onlay graft take satisfactorily but later fracture; and why did the by-pass graft then succeed and later fracture at the lower junction with the host? The grafts took satisfactorily and appeared to be well-nourished. It can be admitted that in the first operation of double onlay graft the screw holes formed a good reason for local weakness; but after there had been fracture through the site of insertion of one screw, why was there no attempt at union?

The underlying principles seem clear to me now and if, after further experience, they are accepted as valid by others, they support McFarland's contention that the by-pass graft offers the greatest hope of success in the treatment of these difficult cases.

Teaching of the anatomy of the limbs gives a fair idea of muscle function in relation to the origin and insertion of muscles and their tendons, and of their action in relation to joints. At first sight, the shape of a limb appears to be determined by the shape of bones and joints, and the muscles covering them; but scant attention has been drawn to the function of the deep fascia and intermuscular septa, except in relation to their special development to serve as pulleys in the neighbourhood of joints. Anatomical dissection shows that the deep fascia of the calf is well developed and has a considerable component of transverse fibres encircling the limb, and it seems clear that the deep fascia and intermuscular septa attached to it serve a definite function in preserving the shape of the limb and controlling the direction of muscular action.

When a deformity such as that shown in Figure 1 has existed for a long period, or has become established in infancy before the shape of the limb has become fixed, bow-stringing of the muscles must take place because the deep fascia is not developed sufficiently to maintain close apposition of the muscles to the bone. Muscle action then produces leverage at the site of fracture which results in a state of affairs that is incompatible with the development of bone-which fortunately is uncommon in adult fractures.

In the case under discussion deformity was long established; and at operation there was found, behind the tibia between the muscles and the bone, a space occupied by a fibro-fatty pad. At the first operation, after dissection and freeing the bone ends, and reducing them in length by stepping so that the tibia could be straightened without difficulty, an onlay graft was applied as a sandwich and the grafts were fixed by screws to maintain a straight and strong but rather shortened tibia. After operation the limb was protected in plaster for a long period. Union took place readily; the grafts and host bone lost their separate characters; and a living tibia reappeared. When the graft was incorporated, relaxation of external splintage was allowed but it was not long before refracture took place and the original deformity recurred. 
This rapid return of deformity was quite unlike that which occurs after ordinary fractures of the middle or lower third of the tibia, and the reason for it was undoubtedly that there was no binding deep fascia to hold the muscle planes close to the tibia. Soon after the bone refractured bow-stringing again took place: a hinge action was set up at the site of fracture and non-union was inevitable.

The by-pass graft was carried out as the only reasonable alternative to repetition of the onlay graft and it is clear from the illustrations that, in some ways, it overcame the disadvantages arising from correction of the original deformity. The fascial attachments to the tibia in its deformed state were not interfered with, and the bone graft was inserted in a

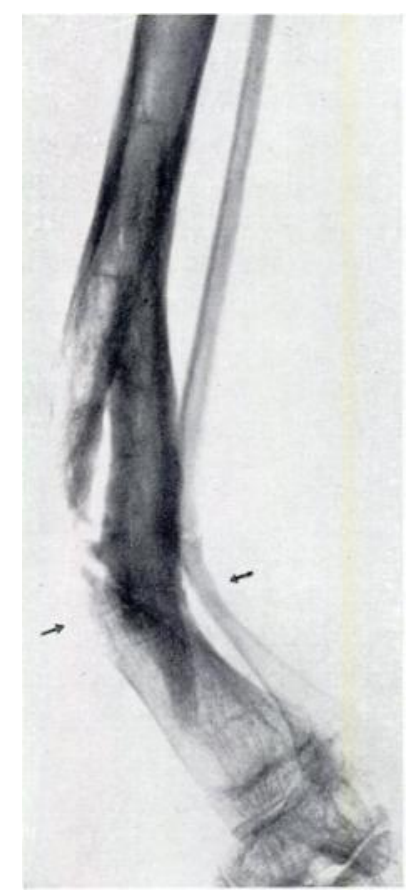

FIG. 1

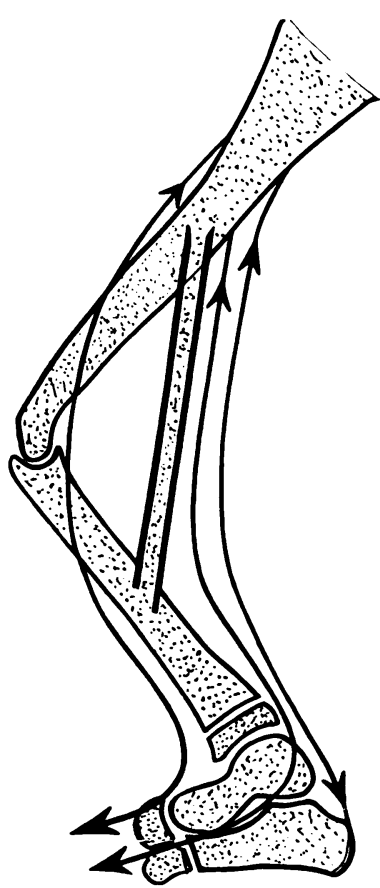

FIG. 2

Figure 1-Refracture at the junction of graft with lower fragment after by-pass grafting for pseudarthrosis of the tibia (same patient as in Case 4, page 40). Figure 2-Diagram to show lines of force in a limb with marked angular deformity. Arrows indicate lines of pull of various muscle groups. Distortion gives rise to bow-stringing of the posterior muscles, and stability can be reached only when a new shaft is formed on the axis of pull of the muscles. Compression forces along the graft are the sum of the components of muscle pull and weight-bearing. In this case the insertion of the graft into the lower fragment was too high and allowed further angulation to take place at the host-graft junction.

plane parallel to the line of muscular action. Its position ensured that it was subjected to longitudinal compression, and close apposition of muscles to the graft was maintained, for the graft lay behind the tibia where the fibro-fatty pad filled the angle between the bone fragments. After insertion of the graft the deep fascia and intermuscular septa were held forwards in the manner of guy ropes, to the deformed tibial shaft. The fascia remained taut; its original function was restored; and it took again its normal part in moulding the shape of the limb. It is clear that if a by-pass graft is to be effective the stress to which it is subjected at each end must be a direct compression force by which to stimulate union. The refracture seen in Figure 1 occurred because the graft was too short and was set too high in the lower 
end of the tibia, so that an angle was formed which was broken by bow-stringing from muscular action. The next stage in repairing the bone must be to lay another bone graft posteriorly, deep to the calf muscles.

Surely, encompassed within the problems that this case presents, must lie the whole key to the persistence of living bone in the human body. It is now realised that living, functioning bone is not a static structure; its texture is always being modified by the process of bone formation and bone removal, and such activity is probably greatest in the natural compression lines of the body where stresses stimulate development of the internal architecture. It may well be that variation in the mechanical stresses imposed on bone is the cause of the continuous modifications that take place, just as it appears to be the cause of modification of bone where strength is impaired-as, for example, in active rickets or Paget's disease where buttressing on the concave side attempts to balance increasing angular deformity. If this is so, the texture of a long bone such as the tibia will probably persist intact only if its general contour is in line with the compression forces imposed upon it by surrounding muscles and weight-bearing. Figure 2 indicates the angle of pull of various muscle groups, the components of which must result, if the shape of the tibia is to remain constant, as a compression strain along the tibial graft and not as a strain exposing the graft to angulation. It is clear from the above discussion that compression stresses are necessary to ensure the integrity of healthy bones and to promote repair after fracture.

Other important factors that can complicate the transition of a fracture-haematoma to the cellular pattern of callus are the surface phenomena that exist between bone, fracturehaematoma and surrounding soft tissues. Bone in contact with other tissues in comparatively short time becomes surfaced by a compact layer within which sheath cancellous bone can achieve maturity from callus. Poor apposition of fragments, inefficient immobilisation and a poorly developed fracture-haematoma, seem to be the causes which allow the development of a similar surface phenomenon between the fragments and lead to non-union. This surface phenomenon is clearly revealed in fractures with angulation, where bone formation can be seen to be deficient on the convex side so that a permanent gap in the surface may result. Periosteum itself appears to have little power to form bone, but all bone surfaces and the surfaces of bone grafts appear to develop a periosteum. The healing of a fracture by bone graft appears to involve quite different principles from the union of a fracture by callus, and it seems likely that the main function of a bone graft, whether compact or cancellous, is to prevent a surface reaction arising between bone and soft tissue that can isolate the two fragments of the bone. The bone fragments and bone graft develop one sheath of compact bone, surfaced by periosteum, within which mechanical strains and stresses can develop the internal architecture.

If these considerations are correct then they help to account for early union of a difficult fracture following a large cancellous bone graft. The packed cancellous bone graft by its mass and its close fit to freshened bony surfaces promotes a surface reaction to the surrounding soft tissues which causes the laying down of a compact layer of bone. Within this the remoulding forces allow the development of living cancellous bone architecture without the development of callus in a fracture-haematoma, the process being comparable to the normal process of absorption and formation of bone constantly occurring in normal bone.

VOL. 33 B, NO. 1, FEBRUARY 1951 